\title{
Editorial
}

\section{Turbulence and Fluid Mechanics}

\author{
Ziemowit Malecha (D)
}

check for

updates

Citation: Malecha, Z. Turbulence and Fluid Mechanics. Energies 2022, 15, 1116. https://doi.org/10.3390/ en15031116

Received: 25 January 2022

Accepted: 29 January 2022

Published: 2 February 2022

Publisher's Note: MDPI stays neutral with regard to jurisdictional claims in published maps and institutional affiliations.

Copyright: (C) 2022 by the author. Licensee MDPI, Basel, Switzerland. This article is an open access article distributed under the terms and conditions of the Creative Commons Attribution (CC BY) license (https:// creativecommons.org/licenses/by/ $4.0 /)$.
Department of Cryogenics and Aerospace Engineering, Wrocław University of Science and Technology, 50-370 Wrocław, Poland; ziemowit.malecha@pwr.edu.pl

\section{Introduction}

This Special Issue of Energies features 11 scientific papers on the subject of turbulence and fluid mechanics. Each of the submitted papers contribute to experimental, numerical and/or theoretical studies.

It is both trivial and true to say that fluid flow and turbulence are ubiquitous, especially when considering that these topics have been studied extensively since ancient times to our present day. This has made the discovery of many important laws related to the physics of fluids possible, while also aiding in the formulation of new questions, as well as the posing of new problems. To date, a great deal of contributions have been made toward the understanding of fluid mechanics and turbulence, but there are still many unanswered questions.

It is worth noting that the 2021 Nobel Prize in Physics was awarded to Syukuro Manebe, Klaus Hasselmann and Giorgio Parisi for their pioneering research on complex systems, climate and weather modeling-topics closely related to fluid mechanics and turbulence itself. The significant advancement of computing machines, in particular, has greatly facilitated the impressive development of computation fluid dynamics. This has made very accurate and detailed modeling of flow problems possible on many scales. At the same time, this has also shown us that today's supercomputers are still too weak to cope with the physics of fluids and turbulence. This results in a constant need to create newer, more efficient algorithms and computational methods, as well as the need to use more intelligent simplifications of real flow problems. So we return, once again, to the above-mentioned Nobel laureates, whom, in the 1960s and 1970s, defined the first simplified models of climate and weather that would become the basis of modern models. Both older and modern flow models require a deep understanding of the fundamentals of fluid mechanics and turbulence. They were, and still are, limited by the capabilities of the computing machines accompanying them. The skillful use of available computing resources and the definition of key aspects of the studied flows remain as important today as they were many decades ago.

The purpose of this Special Issue is to cover a wide range of topics related to turbulence and fluid mechanics and to link these discussions between engineering and science. As guest editor of this issue, I believe that creating a common ground between these topics can only serve to benefit the community and help facilitate meaningful collaboration.

The next chapter provides a brief overview of each article, roughly classifying them as experimental, theoretical and/or computational.

\section{A Short Review of the Contributions in This Issue}

The process of grouping and categorizing scientific articles is not always very straightforward, as some papers may cover both experimental and theoretical, as well as computational, aspects. To avoid referring to the same articles repeatedly, they will be presented one-by-one, along with a brief summary of their content.

The opening article [1], entitled "Effects of an Inlet Vortex on the Performance of an Axial-Flow Pump", studies the trends of changes in the inlet vortex and its impact on the operation of the axial-flow pump. The presented research is both numerical and 
experimental. The experiment included four groups of vortex generators installed upstream of the pump impeller to produce stable vortices at the impeller inlet. The dynamics of changes in the shape of vortices in the impeller and the corresponding pressure fluctuations were revealed. The conducted numerical calculations confirmed that the vortices disturb the inlet flow to the impeller, causing a significant reduction in the axial velocity, the weighted average angle and the uniformity of the axial velocity.

The article [2] entitled "Impact of Chemistry-Turbulence Interaction Modeling Approach on the CFD Simulations of Entrained Flow Coal Gasification" presents numerical studies of the influence of various chemistry and turbulence modeling methods on simulation accuracy. The authors focus on the gasification of coal in entrained-flow reactors. The models considered include infinitely fast chemistry and the eddy dispersion concept, and the effect of turbulence on chemical reactions. The authors paid particular attention to the water-gas shift reaction, which turned out to have a key impact on the final gas composition. The following reactors were analyzed in the study: the pilot-scale Mitsubishi Heavy Industries reactor, laboratory-scale reactor at Brigham Young University and the Conoco-Philips E-gas reactor. The main finding of the paper is that advanced turbulence chemistry models with complex kinetic mechanisms gave the best agreement with experimental data.

The article [3] entitled "A Simplified Method for Modeling of Pressure Losses and Heat Transfer in Fixed-Bed Reactors with Low Tube-to-Particle Diameter Ratio" focuses on modeling fixed bed reactors using porous models. The proposed simplified methodology showed that complicated methods of mapping the internal structure of the bed can be avoided. The studies performed showed that the basic method of the porous medium required some modification to account for channeling, wall effects and local backflows. In addition, the authors introduced an anisotropic thermal conductivity coefficient to correctly reproduce heat transfer in the direction perpendicular to the flow of the primary fluid. The validation of the proposed methodology has shown that it is possible to obtain satisfactory results of the fixed-bed reactor model without using a complicated numerical mesh and long-term calculations.

The article [4] entitled "Collapse of n Point Vortices, Formation of the Vortex Sheets and Transport of Passive Markers" contains theoretical studies related to the motion of the $\mathrm{n}$-vortex system. The main subject of the analysis is the phenomenon of vortices collapsing to a single point in a finite time. The motion of the vortices is described by a system of ordinary differential equations that can be solved analytically. To find the collapse location of the vortices, the corresponding nonlinear algebraic system was solved using the Newton's procedure. The research showed that if there are several vortices of much greater intensity, in the collapsing system of 50-point vortices, the weaker vortices organize themselves into the vortex sheet. The article presents the variety of collapsing structures. It should be emphasized that the phenomenon of the collapse of a large number of vortices and their ability to self-organize is new in the context of point vortex dynamics and may be helpful in a better understanding of turbulence.

The article [5] entitled "CTF and FLOCAL Thermal Hydraulics Validations and Verifications within a Multiscale and Multiphysics Software Development" deals with thermal and hydraulic models for designing nuclear reactors. This is of great importance nowadays, as nuclear energy can play a major role in preventing climate change, and proven models can significantly reduce the costs of designing and optimizing nuclear reactors. The authors managed to show that both the CTF and FLOCAL codes can provide a wide range of nocross-flow, cross-flow and turbulent mixing methods suitable for modeling nuclear reactors.

The article [6] entitled "Simplified Transition and Turbulence Modeling for Oscillatory Pipe Flows", while addressing the well-known problem of turbulent pipe flow, still provides new insight into the problem. The authors focus on the computation of one-dimensional, unsteady, Reynolds-averaged Navier-Stokes equations for oscillatory transient and turbulent pipe flows. Their simulations include a wide range of oscillatory Reynolds and Womersley numbers and an implementation of the unsteady Johnson-King 
model. It has been shown that in the case of a much higher than commonly accepted critical value of oscillatory Reynolds numbers, the fully and continuous turbulence models correspond best with the experimental data.

The article [7] titled "The Thermal-Flow Processes and Flow Pattern in a Pulsating Heat Pipe-Numerical Modelling and Experimental Validation" deals with very challenging and important problems related to multi-phase flows. Additionally, the authors present very interesting and original experiment of a flow in pulsating heat pipe. The corresponding numerical model was based on OpenFoam software and the volume of fluid method (VOF). The numerical calculations were carried out with ethanol being treated as a working medium, and the initial and boundary conditions were taken directly from the experimental measurements. For the analyzed velocity ranges, the relative error, between experimental and numerical results, was at the level of $10 \%$.

The article [8] entitled "Hydrodynamics of Liquid-Liquid Flows in Micro Channels and Its Influence on Transport Properties: A Review" is a review article on heat and mass transport in microchannels, including flow patterns and typical flow regimes in microchannels. The article discusses both experimental and computational research. In addition, the review takes into account the influence of geometric parameters such as the shape, size and material of the channels, as well as the flow velocity, flow coefficient and fluid properties. The authors note that most of the research related to Liquid-Liquid Extraction (LLE) systems was carried out in the slug flow regime and T-junctions, and the numerical simulations coincide with the experimental results with a deviation of up to $20 \%$. Taken together, the authors suggest that AI-based models could help improve the computational prediction of flow patterns for different systems.

The article [9] entitled "Surface Roughness Effects on Flows Past Two Circular Cylinders in Tandem Arrangement at Co-Shedding Regime" focuses on the study of the effect of surface roughness effects on aerodynamic loads and vortex shedding frequency of two circular cylinders in a tandem configuration. In the study, the roughness model was successfully combined with the Lagrangian vortex method using the sub-grid turbulence modeling. The study revealed changing vortex dynamics behavior, drag reduction, intermittence of vortex shedding and wake destruction for certain roughness characteristics. It should be noticed that the study can be of considerable value in a variety of engineering applications where the roughness effect plays a major role.

The article [10] entitled "Vortex Trapping Cavity on Airfoil: High-Order Penalized Vortex Method Numerical Simulation and Water Tunnel Experimental Investigation" presents new possibilities of using the vortex-in-cell (VIC) method to model geometrically complicated flows. The authors use their method to model aerodynamic profiles with various vortex trapping cavities. The authors present in detail the two-dimensional implementation of the high-order penalized VIC method. The work also includes experimental visualizations of the flows under consideration. The obtained results show that the developed numerical methodology is valuable in the case of highly swirling flows where vorticity plays a major role and that the aerodynamic performance of airfoils can be improved by using a trapping vortex cavity.

The article [11] titled "Numerical study of baroclinic acoustic streaming phenomenon for various flow parameters" presents a numerical study of the large-amplitude, acousticallydriven streaming flow for different frequencies of the acoustic wave and different temperature gradients between hot and cold surfaces. The study includes two-dimensional rectangular resonators of different lengths, one three-dimensional rectangular resonator and one long and narrow channel, representative of a typical U-shaped resistance thermometer. The research confirmed that the baroclinic acoustic streaming was largely independent of the frequency, and its intensity increased with the temperature gradient between the hot and cold surface. It should be noted that a slight maximum was observed for some oscillation frequencies. The main novelty is the 3D calculations, which revealed the formation of a three-dimensional pair of streaming vortices. Furthermore, the authors showed that the 
intensity of streaming was gradually decreasing while approaching the side walls of the resonator and created a quasi-parabolic profile.

\section{Conclusions}

The papers featured in this Special Issue of Energies cover a wide range of topics related to turbulence and fluid mechanics. These range from a theoretical analysis of the dynamics of point vortices to multiphase flow experiments, including simulations of nuclear reactors. Each article contributes to theoretical, numerical and/or experimental studies. Presenting this broad range of topics together in one publication can help highlight the inherent link between engineers and scientists from various fields and enhance their multidisciplinary insights.

Funding: This research received no external funding.

Conflicts of Interest: The author declares no conflict of interest.

\section{References}

1. Zhang, W.; Tang, F.; Shi, L.; Hu, Q.; Zhou, Y. Effects of an Inlet Vortex on the Performance of an Axial-Flow Pump. Energies 2020, 13, 2854. [CrossRef]

2. Mularski, J.; Modliński, N. Impact of Chemistry-Turbulence Interaction Modeling Approach on the CFD Simulations of Entrained Flow Coal Gasification. Energies 2020, 13, 6467. [CrossRef]

3. Świeboda, T.; Krzyżyńska, R.; Bryszewska-Mazurek, A.; Mazurek, W.; Wysocka, A. A Simplified Method for Modeling of Pressure Losses and Heat Transfer in Fixed-Bed Reactors with Low Tube-to-Particle Diameter Ratio. Energies 2021, 14, 784. [CrossRef]

4. Kudela, H. Collapse of $\mathrm{n}$ Point Vortices, Formation of the Vortex Sheets and Transport of Passive Markers. Energies 2021, 14, 943. [CrossRef]

5. Davies, S.; Rohde, U.; Litskevich, D.; Merk, B.; Bryce, P.; Levers, A.; Detkina, A.; Atkinson, S.; Ravindra, V. CTF and FLOCAL Thermal Hydraulics Validations and Verifications within a Multiscale and Multiphysics Software Development. Energies 2021, 14, 1220. [CrossRef]

6. Shapiro, A.; Grossman, G.; Greenblatt, D. Simplified Transition and Turbulence Modeling for Oscillatory Pipe Flows. Energies 2021, 14, 1410. [CrossRef]

7. Błasiak, P.; Opalski, M.; Parmar, P.; Czajkowski, C.; Pietrowicz, S. The Thermal—Flow Processes and Flow Pattern in a Pulsating Heat Pipe-Numerical Modelling and Experimental Validation. Energies 2021, 14, 5952. [CrossRef]

8. Ganguli, A.A.; Pandit, A.B. Hydrodynamics of Liquid-Liquid Flows in Micro Channels and Its Influence on Transport Properties: A Review. Energies 2021, 14, 6066. [CrossRef]

9. Moraes, P.G.d.; Alcântara Pereira, L.A. Surface Roughness Effects on Flows Past Two Circular Cylinders in Tandem Arrangement at Co-Shedding Regime. Energies 2021, 14, 8237. [CrossRef]

10. Błoński, D.; Strzelecka, K.; Kudela, H. Vortex Trapping Cavity on Airfoil: High-Order Penalized Vortex Method Numerical Simulation and Water Tunnel Experimental Investigation. Energies 2021, 14, 8402. [CrossRef]

11. Baran, B.; Machaj, K.; Malecha, Z.; Tomczuk, K. Numerical Study of Baroclinic Acoustic Streaming Phenomenon for Various Flow Parameters. Energies 2022, 15, 854. [CrossRef] 